\title{
Welt-Thrombosetag 2017
}

Der mittlerweile 4. Weltthrombosetag fand am 13.10. 2017 erneut im Allianzforum in Berlin mit wunderbarem Blick auf das Brandenburger Tor und auf den Pariser Platz statt.

Federführend für die Auswahl des Mottos „Knoten lösen, Leben retten - intersektorale Versorgung der Thrombose und Lungenembolie" und die Konzeption des hochkarätig besetzten Podiums waren die Vertreter der beteiligten Gefäßgesellschaften: DGP - Dr. Jutta Schimmelpfennig,
DGA - Prof. Dr. Rupert Bauersachs (wissenschaftliche Leitung „Aktionsbündnis Thrombose“), GTH - Prof. Dr. Bettina Kemkes-Matthes, Deutsche Gefäßliga - PD Dr. Christoph Kalka.

Unter Leitung der Medizinjournalistin Lisa Braun wurde die unverändert bestehende Problematik der noch nicht optimal organisierten Versorgung von Patienten mit Thrombose aus verschiedenen Blickwinkeln thematisiert. Zwar sei die Diagnostik und auch die Therapie der Throm-
Abb. 1

Teilnehmer des Weltthrombosetags

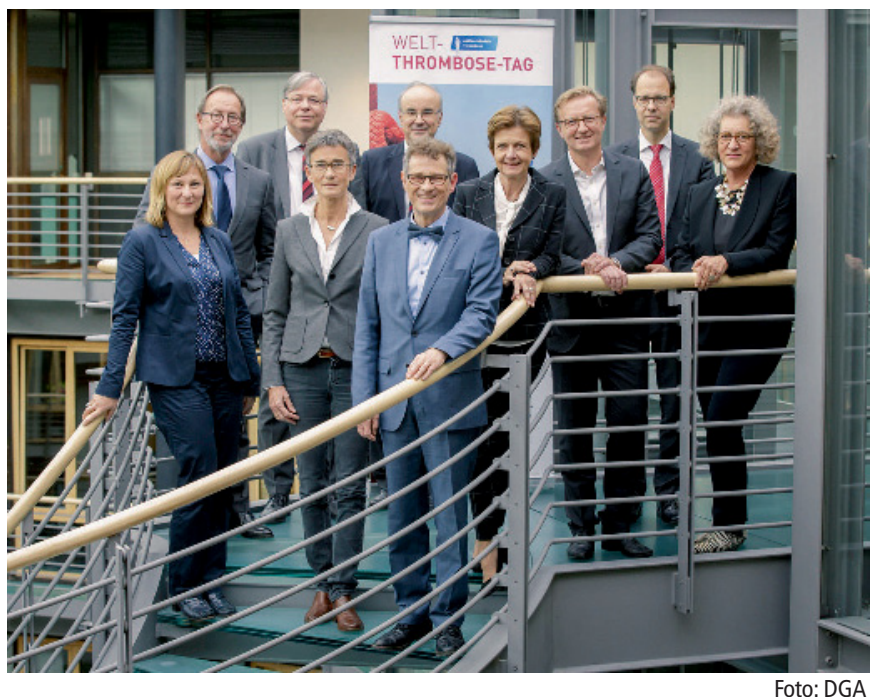

bose durch die aktuellen Leitlinien den Einsatz moderner Antikoagulantien und eine symptomorientierte Kompressionstherapie zielgerichteter und dadurch risikoärmer geworden, trotzdem beständen nach wie vor Defizite im zeitgerechten Beginn der Therapie, wie auch im Ansatz der Behandlungsdauer.

Dies würde sowohl zur Unter- wie auch zur Überversorgung führen können.

Um diese Defizite auszugleichen wurde die „Antikoagulations-Ampel“ vorgestellt, ein übersichtliches Schema, welches im "Schreibtischformat" dem behandelnden Arzt wesentliche Entscheidungshilfen zur individuellen Therapiedauer und damit zur Vermeidung einer Überversorgung eines Thrombosepatienten geben kann.

In Bezug auf die Unterversorgung wurde darauf hingewiesen, dass seit der „Tulipa-Studie“ 2005 keine aktuellen objektiven Daten zur Versorgungsrealität von Patienten mit Thromose oder Lungenembolie in der Bundesrepublik bestünden.

Frau Dr. Regina Klakow-Franck, unparteiisches Mitglied des gemeinsamen Bundesausschusses (G-BA), empfahl darauf hin, eine industrieunabhängige, flächendeckende Versorgungsstudie zur Versorgungsrealität $\mathrm{zu}$ initiieren und bald möglichst einen Förderantrag an den Innovationsfond des Gesundheitsministeriums zu stellen.

\section{Dr. Jutta Schimmelpfennig}

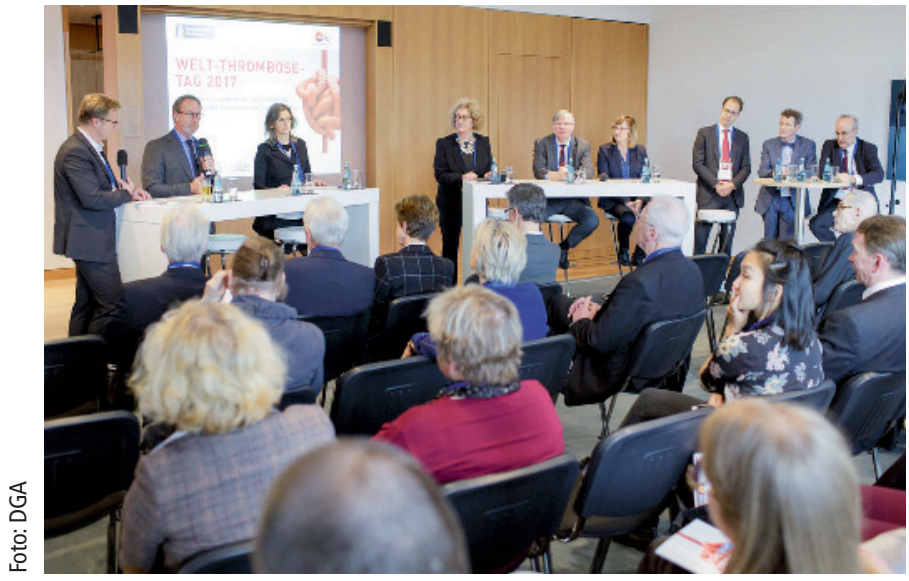

Abb. 2 Veranstaltung im Rahmen des Weltthrombosetags

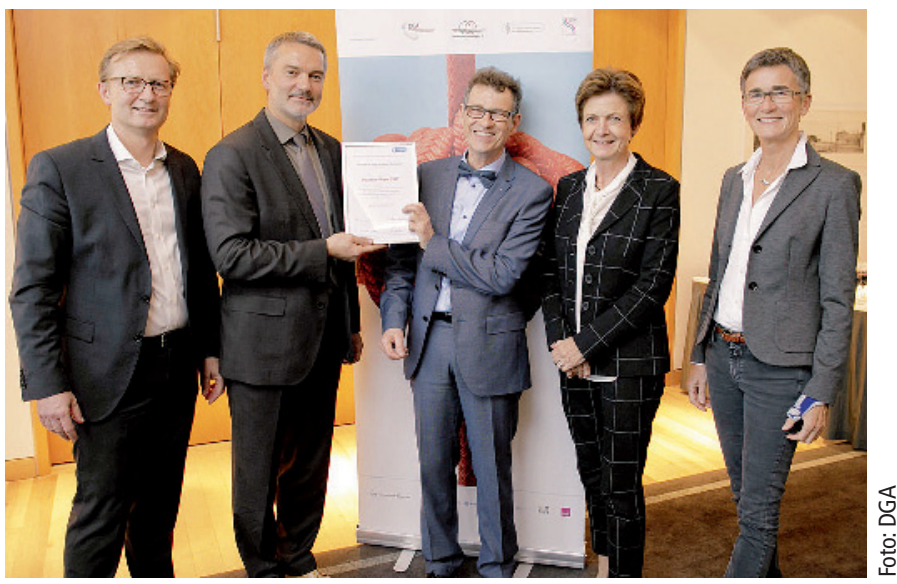

Abb. 3 Verleihung des Virchow-Preises 2017 für das „Dresdner NOAK-Register" durch das "Aktionsbündnis Thrombose" anlässlich des Weltthrombosetages in Berlin an PD Dr. Jan Beyer-Westendorf: PD Dr. Christoph Kalka, PD Dr. Jan Beyer-Westendorf, Prof. Dr. Rupert Bauersachs, Dr. Jutta Schimmelpfennig, Prof. Dr. Bettina Kemkes-Matthes (v.l.n.r) 


\section{Jahrestagung der DGP in Bielefeld}

Unter dem Motto „Auch Heldinnen haben (kranke) Beine" findet unter der wissenschaftlichen Leitung von PD Dr. Helger Stege die 60. Jahrestagung unserer Gesellschaft in Bielefeld statt.

Das Motto: „Auch HeldInnen haben (kranke) Beine" bezieht sich auch auf den Ort der Tagung nahe des Teutoburger Waldes, in dem Armin / Hermann die berühmte Varusschlacht gegen die Römer geschlagen hat.

Das Motto weist aber auch auf die phlebologischen und lymphologischen Patientinnen und Patienten hin, die teils heldenhaft die Einschränkungen ihres Lebens durch Leiden aus dem phlebologischen Formenkreis meistern. Dank modernster Verfahren in Diagnostik, Behandlung, Vorund Nachsorge gelingt es, interdisziplinär, interprofessionell und mit Hilfe der Entwicklungen der Industrie unseren Patienten vielfach Heilung, fast immer Besserung der Symptome oder Hilfe in der Bewältigung der Erkrankungen anzubieten. Dies geschieht leitliniengerecht aber auch unter individuellen Gesichtspunkten mit viel Herz, Verstand und Erfahrung.

Der interdisziplinäre Austausch im Rahmen der Jahrestagung wird gewährleistet durch die Teilnahme anderer wissenschaftlicher Fachgesellschaften wie der DGA, der DGG, DGL und der DGfW/ ICW. Die Interprofessionalität spiegelt sich in gemeinsamen Sitzungen mit Pflegekräften und medizinischen Fachangestellten wider. Die 60. Jahrestagung der DGP wird örtlich sehr konzentriert in der Stadthalle Bielefeld stattfinden. Inhaltlich wird das Programm einen weiten Bogen spannen. Klassische phlebologische Themen wie Kompressionstherapie, Sklerosierungsbehandlungen und endovenöse oder operative erfahren finden ihren angestammten Platz ebenso wie die Beantwortung von Fragen zu Thrombose, Thromboembolie, Lymphödem und zu Erkrankungen, die der Phlebologe differentialdiagnostisch im Blick behalten sollte. Behandlung chroni-

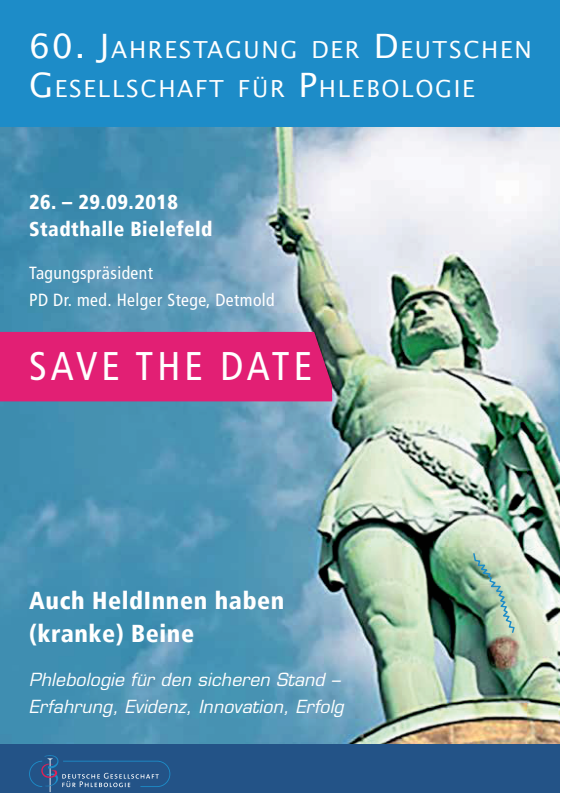

Abb. 4 Jahrestagung der DGP 2018 in Bielefeld

scher Wunden und Ernährung sind wichtige Themen, die weitere Schwerpunkte bilden. Nähere Informationen erhalten Sie über die Website zur Tagung https://phlebologie-2018.de/

\section{Bayerische Staatsmedaille für Verdienste um Gesundheit und Pflege in Nürnberg ver- liehen}

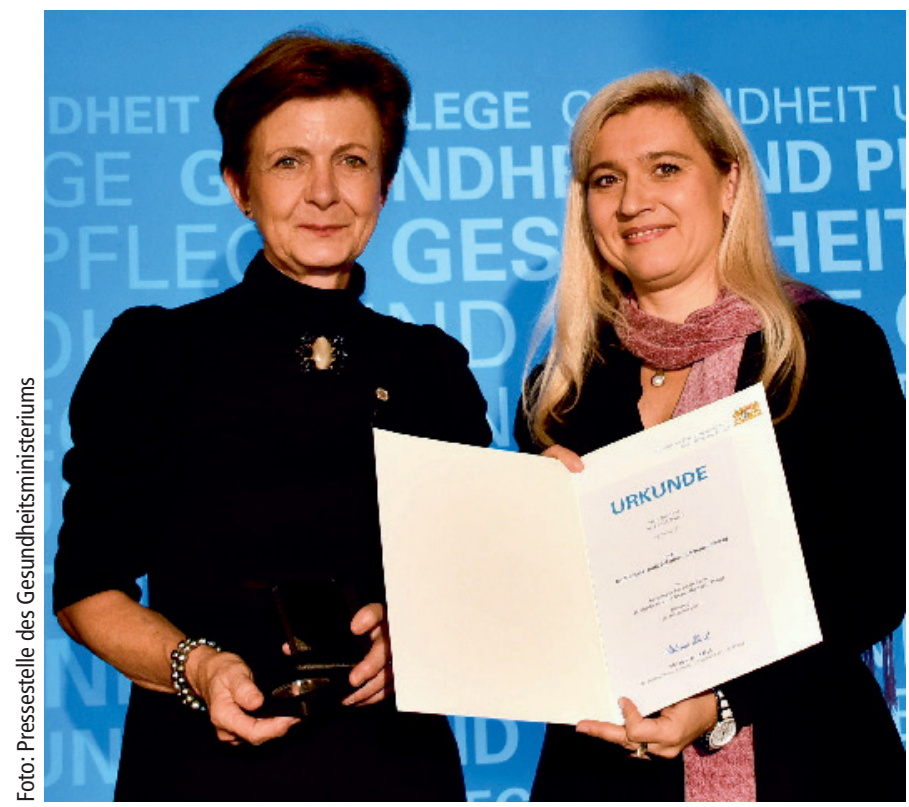

Abb. 5

Bayerns Gesundheitsund Pflegeministerin Melanie Huml (rechts) verleiht die Bayerische Staatsmedaille für Verdienste um Gesundheit und Pflege an Frau Dr. Jutta Schimmelpfennig
Bayerns Gesundheits- und Pflegeministerin Melanie Huml hat zehn engagierte Bürgerinnen und Bürger mit der Bayerischen Staatsmedaille für Verdienste um Gesundheit und Pflege ausgezeichnet. Huml betonte anlässlich der feierlichen Verleihung in Nürnberg: „Als Zeichen des Dankes und der Anerkennung für herausragendes Engagement verleiht das bayerische Gesundheits- und Pflegeministerium die Staatsmedaille für Verdienste um Gesundheit und Pflege. Die heute Geehrten sind als Vordenker und Menschen der Tat ein Vorbild. Die Medaille soll für andere Menschen Ansporn sein zu eigenen Anstrengungen. Sie soll ermutigen, zum Wohle der Gesellschaft neue Wege im Bereich der Gesundheit und Pflege zu gehen."

Die Staatsmedaille für Verdienste um Gesundheit und Pflege ist die höchste Auszeichnung des bayerischen Gesundheitsund Pflegeministeriums. Es werden damit Personen geehrt, die sich langjährig ehrenamtlich im Bereich Gesundheit und Pflege engagiert haben. 
In diesem Jahre hat u.a. Frau Dr. Jutta Schimmelpfennig für ihr Engagement für die medizinische Versorgung insbesondere im Bereich der Gefäßerkrankungen die Medaille erhalten. Die Ministerin unterstrich: „Frau Dr. Schimmelpfennig hat neben ihrer ärztlichen Tätigkeit in hohem Maß Zeit und Kraft für die medizinische Versorgung der Bevölkerung aufgewendet. Seit 1989 ist sie als Vorsitzende der Sektion
Bayern-Nord des Deutschen Ärztinnenbundes tätig. Seit 2010 ist Frau Dr. Schimmelpfennig als 1 . Vorsitzende des Vereins Gesundheitsregion Bamberg aktiv und unterstützt die in der Region tätigen Experten aus den Bereichen Gesundheitsversorgung, Gesundheitswirtschaft und -Forschung. Seit 2009 ist sie außerdem Mitglied im Vorstand beziehungsweise Beirat der Deutschen Gesellschaft für Phlebologie und seit
2010 zudem 2. Vorsitzende des Berufsverbandes der Phlebologen. In diesen Funktionen nimmt sie eine wichtige Mittlerrolle für die ärztlichen Kollegen zu berufspolitischen Themen aus dem Fachbereich Gefäßerkrankungen ein."

Verantwortlich für Mitteilungen der DGP: Dr. med. Erika Mendoza 